\title{
Video Article \\ Determining the Chemical Composition of Corrosion Inhibitor/Metal Interfaces with XPS: Minimizing Post Immersion Oxidation
}

\author{
Monika S. Walczak ${ }^{1}$, Perla Morales-Gil ${ }^{1,2}$, Turia Belashehr ${ }^{1}$, Kiran Kousar ${ }^{1}$, Paulina Arellanes Lozada ${ }^{1,3}$, Robert Lindsay ${ }^{1}$ \\ ${ }^{1}$ Corrosion and Protection Centre, School of Materials, The University of Manchester \\ ${ }^{2}$ Laboratorio de Caracterización de Materiales Sintéticos y Naturales, Instituto Mexicano del Petróleo \\ ${ }^{3}$ Departamento de Metalurgia y Materiales, Instituto Politécnico Nacional
}

Correspondence to: Robert Lindsay at robert.lindsay@manchester.ac.uk

URL: https://www.jove.com/video/55163

DOI: doi:10.3791/55163

Keywords: Chemistry, Issue 121, X-ray photoelectron spectroscopy, corrosion inhibition, acidic solution, metallic substrate, oxidation, inert atmosphere

Date Published: 3/15/2017

Citation: Walczak, M.S., Morales-Gil, P., Belashehr, T., Kousar, K., Arellanes Lozada, P., Lindsay, R. Determining the Chemical Composition of Corrosion Inhibitor/Metal Interfaces with XPS: Minimizing Post Immersion Oxidation. J. Vis. Exp. (121), e55163, doi:10.3791/55163 (2017).

\section{Abstract}

An approach for acquiring more reliable X-ray photoelectron spectroscopy data from corrosion inhibitor/metal interfaces is described. More specifically, the focus is on metallic substrates immersed in acidic solutions containing organic corrosion inhibitors, as these systems can be particularly sensitive to oxidation following removal from solution. To minimize the likelihood of such degradation, samples are removed from solution within a glove box purged with inert gas, either $\mathrm{N}_{2}$ or Ar. The glove box is directly attached to the load-lock of the ultra-high vacuum Xray photoelectron spectroscopy instrument, avoiding any exposure to the ambient laboratory atmosphere, and thus reducing the possibility of post immersion substrate oxidation. On this basis, one can be more certain that the X-ray photoelectron spectroscopy features observed are likely to be representative of the in situ submerged scenario, e.g. the oxidation state of the metal is not modified.

\section{Video Link}

The video component of this article can be found at https://www.jove.com/video/55163/

\section{Introduction}

Corrosion inhibitors (Cls) are substances that, when introduced into an aggressive environment, reduce the corrosion rate of a metallic material by inducing a change at the solid/liquid interface ${ }^{1,2,3,4,5}$. This approach to corrosion control is widely used in industry, with high performance Cls having been successfully developed for a variety of applications. There remains, however, a considerable lack of fundamental understanding of $\mathrm{Cl}$ performance, hindering knowledge-based optimization. For example, the precise nature of interfaces formed by organic-Cls in corrosive acidic solutions is still unclear.

Given the assumption that organic-Cls inhibit acidic corrosion through formation of an adsorbed 2-D layer ${ }^{2}$, a surface sensitive technique is required to characterize these interfaces. Consequently, X-ray photoelectron spectroscopy (XPS) ${ }^{6}$ has emerged as a technique of choice to probe the elemental/chemical composition of these interfaces ex situ ${ }^{7,8,9} ;$ XPS measurements are typically performed in, or close to, ultra-high vacuum (UHV). Various insights have been claimed, including that a surface oxide or hydroxide is present to facilitate organic-Cl binding to the metallic substrate ${ }^{10,11,12}$. The validity of this interface description, however, is questionable as the XPS data were acquired from samples that had been exposed to the laboratory atmosphere in between removal from inhibited solution and introduction into the UHV-XPS spectrometer. Such a procedure may result in interface oxidation, undermining conclusions about interface chemical composition. An alternative approach is required, which minimizes the potential for post immersion oxidation.

In this paper, we detail a methodology designed to allow one to acquire XPS data from organic-Cl/metal interfaces that have not undergone oxidation following emersion from acidic solution. A glove box, purged with inert gas, which is attached directly to the vacuum load-lock of the UHV-XPS instrument is employed. The utility of our approach is verified through presentation of XPS data from two organic-Cl/carbon-steel interfaces formed following addition of sufficient $\mathrm{Cl}$ to significantly reduce the substrate corrosion rate in aqueous $1 \mathrm{M} \mathrm{hydrochloric} \mathrm{acid}(\mathrm{HCl})$ solution.

\section{Substrate/Solution Preparation}

\section{Preparation of carbon-steel substrate}


1. Cut an approximately $2-3 \mathrm{~mm}$ thick disc from a cylindrical carbon-steel rod ( $10 \pm 2 \mathrm{~mm}$ diameter), using a precision cutting machine. Employ cooling fluid during the cutting procedure to minimize mechanical damage.

2. Grind both circular faces, and the edge of the disc-shaped sample with a series of SiC papers, i.e. 600 grit, 800 grit, 1,200 grit, 2,400 grit, and 4,000 grit.

3. Polish both circular faces, and the edge of the disc-shaped sample with either diamond paste or alumina powder ( $3 \mu \mathrm{m}$ and/or $1 \mu \mathrm{m})$ until a mirror finish is obtained.

4. Sonicate the sample at room temperature for approximately 10 minutes in each of the following: deionized water, acetone, ethanol, and finally deionized water again.

5. Dry all surfaces of the sample under a flow of air.

6. Store the sample in either a desiccator or vacuum desiccator until required. Ideally, finish preparing the carbon-steel substrate just prior to solution immersion.

\section{Preparation of inhibited $1 \mathrm{M} \mathrm{HCl}$ solution}

1. Add $10.2 \mathrm{M} \mathrm{HCl}$ to deionized water to produce a $1 \mathrm{M} \mathrm{HCl}$ solution. CAUTION: $\mathrm{HCl}$ is harmful and corrosive. Undertake this step in a fume hood, wearing suitable personal protection equipment.

2. Dissolve $\mathrm{xg}$ of selected organic- $\mathrm{Cl}$ (e.g. $\mathrm{x}=0.300 \mathrm{~g}$ of 2-mercaptobenzimidazole) in $1 \mathrm{M} \mathrm{HCl}$ solution to produce a $1 \mathrm{M} \mathrm{HCl}$ solution containing $\mathrm{x} \mathrm{mM}$ organic- $\mathrm{Cl}$ (e.g. $\mathrm{x}=2 \mathrm{mM}$ 2-mercaptobenzimidazole).

\section{Substrate Immersion in Inhibited Acid Solution}

1. Pour a small volume (typically $25-50 \mathrm{~mL}$ ) of the $1 \mathrm{M} \mathrm{HCl}+\mathrm{x} \mathrm{mM}($ e.g. $\mathrm{x}=2 \mathrm{mM}$ 2-mercaptobenzimidazole) organic-Cl solution into a small glass beaker.

2. Pick up the disc-shaped carbon-steel sample with either ceramic or plastic tweezers that are resistant to $1 \mathrm{M} \mathrm{HCl}$. Only touch the edge of the sample with the tweezers.

3. Introduce the sample into the glass beaker containing the $1 \mathrm{M} \mathrm{HCl}+\mathrm{x} \mathrm{mM}$ organic- $\mathrm{Cl}$ solution. Orient the sample so that cylindrical faces are in the vertical plane. Deposit the sample either directly onto the bottom of the beaker, or atop the open end of a short glass tube. Ensure that the sample is fully submerged.

\section{Sample Transfer}

1. Glove box preparation

1. Locate the circular sample-transfer opening in the base of the glove box onto the XPS load-lock flange mounting. Ensure that the seal between glove box and load-lock flange is formed correctly.

2. Connect the glove box to an inert gas cylinder (either $\mathrm{N}_{2}$ or $\mathrm{Ar}$ ).

3. Adhere a small square of double-sided carbon (conductive) tape onto the XPS sample bar.

4. Insert hardware required for sample transfer into XPS instrument through an open port on the glove box, i.e. sample bar with carbon tape attached, plastic/ceramic tweezers, nitrile gloves, laboratory tissues, plastic paraffin film, empty/dry wash bottle with spout, and glass beaker containing $\sim 200 \mathrm{~g}$ of $\mathrm{Na}_{2} \mathrm{CO}_{3}$ powder.

5. Place glass beaker containing carbon-steel sample in $1 \mathrm{M} \mathrm{HCl}+\mathrm{x} \mathrm{mM}$ (e.g. $\mathrm{x}=2 \mathrm{mM}$ 2-mercaptobenzimidazole) organic-Cl solution inside glove box. Ensure that sample is always fully immersed during this step.

6. Seal all ports/entry points on the glove box and begin to purge with $\mathrm{N}_{2}$ (or $\mathrm{Ar}$ ).

7. Continuously purge the glove box until the sample transfer is complete.

2. From solution to XPS analysis chamber

1. Allow the sample to remain submerged in the $1 \mathrm{M} \mathrm{HCl}+\mathrm{x} \mathrm{mM}$ organic-Cl solution for the desired immersion period, e.g. $4 \mathrm{~h}$, as employed for the XPS data presented below.

2. Check that the relative humidity within the glove box is minimized, which is typically achieved after $60-90$ min of initiating purging. A value of $8 \%$ relative humidity is required before proceeding with sample transfer; value prior to purging is typically $35-40 \%$. NOTE: Normally, there is no dedicated $\mathrm{O}_{2}$ sensor within the glove box, but trial measurements with such a device indicate that the purging procedure leads to a reduction in $\mathrm{O}_{2}$ concentration by a factor of $\sim 1,000$.

3. Introduce hands into glove box gloves, and then cover gloved hands with nitrile gloves located within the glove box. This step reduces the possibility of contamination during sample handling, as well as increases the ease of sample manipulation.

4. Remove the carbon-steel sample from the $1 \mathrm{M} \mathrm{HCl}+\mathrm{x} \mathrm{mM}$ (e.g. $\mathrm{x}=2 \mathrm{mM}$ 2-mercaptobenzimidazole) organic-Cl solution, using the ceramic/plastic tweezers. Only touch the edge of the sample with the tweezers.

5. Immediately after emersion, blow the sample dry by directing a stream of inert gas, produced by repeatedly squeezing empty/dry wash bottle located inside glove box, onto sample surfaces.

NOTE: Rinsing with solvents at this stage is not undertaken to minimize the potential of damaging the inhibited interface, e.g. inhibitor removal or interface oxidation.

6. Cover beaker containing $1 \mathrm{M} \mathrm{HCl}+\mathrm{x} \mathrm{mM}$ organic-Cl solution with plastic paraffin film.

7. Affix the sample to the small square of double-sided carbon (conductive) tape attached to XPS sample bar. Do not touch the surface to be probed by XPS.

8. Vent the XPS load-lock chamber to $\mathrm{N}_{2} /$ Ar. Ensure that the associated turbo/rotary pump combination is switched off before performing this action.

9. Open the load lock flange.

10. Transfer the sample bar into the load-lock chamber and slide it onto sample holding prong.

11. Close the load-lock flange.

12. Switch on turbo/rotary pump combination to pump down load-lock chamber. 
13. Once pressure in load-lock chamber reaches at least $\sim 5 \times 10^{-7}$ mbar, manually transfer sample to intermediate chamber using transfer arm.

14. Wait until pressure in intermediate chamber reaches $\sim 1 \times 10^{-8} \mathrm{mbar}$, and then use second transfer arm to manually transfer sample onto the sample manipulator in the analysis chamber.

\section{Acquisition of XPS Data}

1. Orient angle of sample, using keypad to drive sample manipulator motors, to desired photoelectron emission angle, e.g. $0^{\circ}$ (emission along the surface normal), as employed for the XPS data presented below.

2. Open XPS data acquisition software by clicking on the icon located on the desktop. Open Instrument Manual Control window.

3. Input $10 \mathrm{~mA}$ and $15 \mathrm{kV}$ as the values for the anode emission and anode HT parameters, respectively. Then, click the "ON" button in the "Xray Gun" section to power up the monochromated Al Ka X-ray source. After this, click the "ON" button in the "Neutralizer" section to turn on the charge neutralizer.

4. Select electron analyzer 'Spectrum/Hybrid' measurement mode from the 'Mode' and 'Lens' drop down menus in the 'Analyzer' section.

5. Input desired kinetic/binding energy ranges, pass energy(ies), step size(s), and dwell time(s) into 'Acquisition/Scan Control' section. Example input parameter values: 1,200 - $0 \mathrm{eV}$ binding energy range, $80 \mathrm{eV}$ pass energy, $0.5 \mathrm{eV}$ step size, and $0.1 \mathrm{~s}$ dwell time to acquire overview spectra.

6. Optimize sample position by adjusting position of sample manipulator, using keypad to drive sample manipulator motors, to maximize signal from selected core level, e.g. C 1s or Fe 2p.

7. Acquire XPS spectra by initiating data collection in XPS data acquisition software.

\section{Representative Results}

Figure 1 shows overview, $\mathrm{O}$ 1s, and Fe 2p XPS data acquired from carbon-steel samples that have been immersed for $4 \mathrm{~h}$ in one of two different $1 \mathrm{M} \mathrm{HCl}+\mathrm{x} \mathrm{mM}$ organic-Cl solutions, and transferred for XPS measurement as detailed above. Analogous data from a polished sample are also displayed. The carbon-steel possessed a nominal weight\% composition of $\mathrm{C}(0.08-0.13), \mathrm{Mn}(0.30-0.50), \mathrm{P}(0.04), \mathrm{S}(0.05)$, and Fe (balance). The two organic-Cls studied are: 2-mercaptobenzimidazole (MBI) and (Z)-2-2(2-(octadec-9-en-1-yl)-4,5-dihydro-1H-imidazole-1-yl)ethanamine (OMID). At the concentrations employed (MBI: $2 \mathrm{mM}$; OMID: $1 \mathrm{mM}$ ) corrosion rate measurements ${ }^{13,14}$ indicate that all of the species significantly inhibit the corrosion of carbon-steel, i.e. inhibition efficiencies $(\eta \%)^{2}>90 \%$. Best fits to the $01 \mathrm{~s}$ and Fe $2 p$ spectral profiles are also displayed. Photoelectron peaks were modeled with Gaussian-Lorentzian (GL) line shape functions (30 \% Lorentzian), with the exception of the Fe $2 p$ level of metallic iron, where a Lorentzian asymmetric line shape with tail damping (LF) was used. The cationic Fe ${ }^{x+}$ states were modeled with multiplet envelopes consisting of 3 and $4 \mathrm{GL}$ functions for $\mathrm{Fe}^{2+}$ and $\mathrm{Fe}^{3+}$, respectively ${ }^{15}$. A Shirley-type function ${ }^{16}$ was used to describe the background of inelastically scattered electrons.

Focusing on the overview XPS data (Figure 1 (a)), the spectrum acquired from the polished sample exhibits three prominent peaks, i.e. Fe $2 p$, $\mathrm{O} 1 \mathrm{~s}$, and $\mathrm{C}$ 1s. These features can be assigned as follows: Fe $2 p$ arises from the carbon-steel, $\mathrm{O} 1 \mathrm{~s}$ derives from both an surface oxidic film and adsorbates, and the $\mathrm{C}$ 1s signal is due to adventitious carbon. Submersion in any of the $1 \mathrm{M} \mathrm{HCl}+\mathrm{x}$ mM organic-Cl solutions results in significant changes to the corresponding overview spectrum. A feature assigned to the $\mathrm{N} 1 \mathrm{~s}$ core level appears, which is consistent with surface adsorption of the inhibitors; MBI and OMID both contain N. Moreover, the O1s core level signal is significantly diminished.

As regards $\mathrm{O} 1 \mathrm{~s}$ data from the polished substrate (Figure 1 (b)), the profile can be fitted with four components. The two components at lower binding energies $(\mathrm{BE}), \sim 530.0 \mathrm{eV}$ and $\sim 531.3 \mathrm{eV}$, can be assigned to iron oxide $\left(\mathrm{O}^{2-}\right)$ and hydroxide $\left(\mathrm{OH}^{-}\right)$phases, respectively. The two higher binding energy components, labeled $\mathrm{O}^{1}(\mathrm{BE} \sim 532.2 \mathrm{eV})$ and $\mathrm{O}^{2}(\mathrm{BE} \sim 533.3 \mathrm{eV})$, are likely associated with adsorbed $\mathrm{OH}\left(\mathrm{O}^{1}\right)$ and adventitious carbon species $\left(\mathrm{O}^{1} \text { and } \mathrm{O}^{2}\right)^{17}$. Immersion in either of the $1 \mathrm{M} \mathrm{HCl}+x \mathrm{mM}$ organic-Cl solutions leads to the complete quenching of the $\mathrm{O}^{2-}$ and $\mathrm{OH}^{-}$components. On this basis, it can be concluded that the corrosion inhibitors are adsorbed on oxide/hydroxide free surfaces. The Fe 2p spectra in Figure 1 (c) are consistent with this result, as only a metallic $\mathrm{Fe}\left(\mathrm{Fe}^{0}\right)$ peak is apparent on the inhibited substrates. $\mathrm{Fe}^{2+}$ and $\mathrm{Fe}^{3+}$ features are present on the polished sample, due to the surface oxide/hydroxide.

$\mathrm{O}$ 1s and Fe 2p core level XPS spectra from two carbon-steel samples immersed in $1 \mathrm{M} \mathrm{HCl}+2 \mathrm{mM} \mathrm{MBI}$ are compared in Figure 2. One sample was transferred using a fully $\mathrm{N}_{2}$-purged glove box, whilst the other was removed from solution into a partially $\mathrm{N}_{2}$-purged glove box, i.e. $\mathrm{O}_{2}$ concentration was significantly higher than target value. For the latter sample, it is apparent that post immersion oxidation has occurred, i.e. $\mathrm{Fe}^{2+/ 3+}$ and $\mathrm{O}^{2-} / \mathrm{OH}^{-}$features are present. 

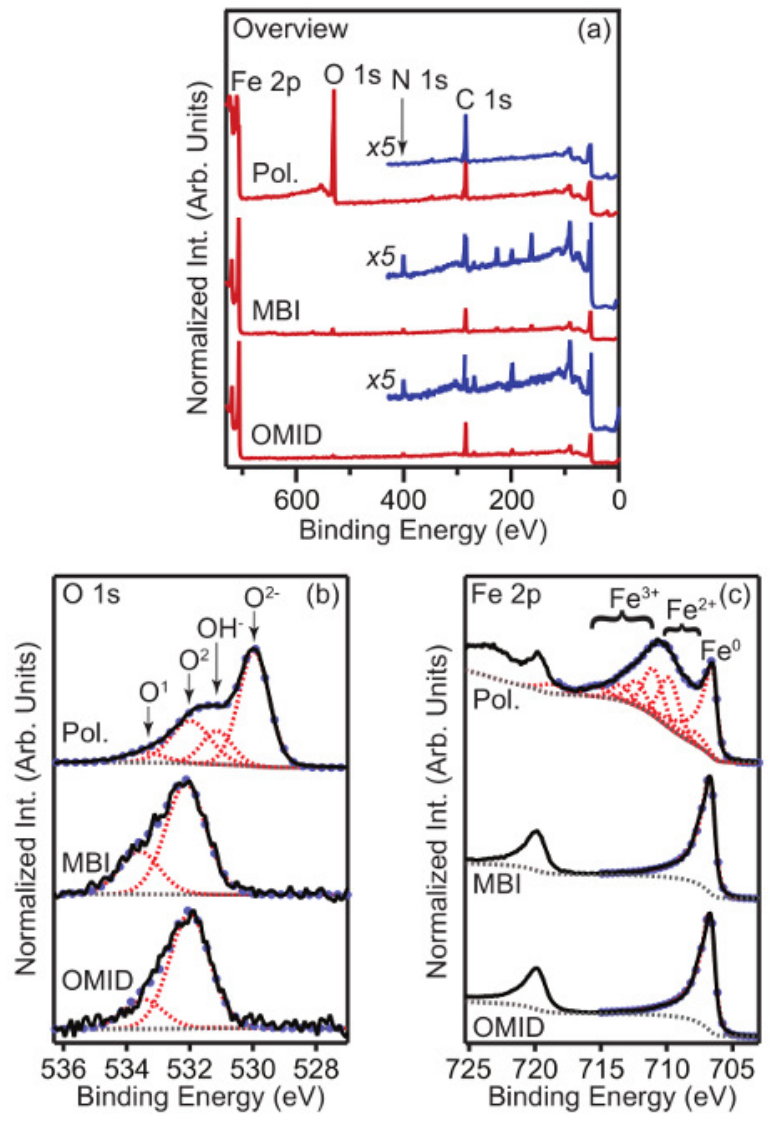

Figure 1. XPS spectra from polished and inhibited carbon-steel samples. (a) Overview, (b) $O$ 1s, and (c) Fe $2 p$ XPS spectra. Data presented in each panel were acquired from carbon-steel samples that had been immersed for $4 \mathrm{~h}$ in one of two different $1 \mathrm{M} \mathrm{HCl}+\mathrm{x} \mathrm{mM}$ organic-Cl solutions, i.e. $2 \mathrm{mM} \mathrm{MBI}$, and $1 \mathrm{mM}$ OMID. Spectra were all acquired at a photoelectron emission angle $\left(\theta_{\mathrm{E}}\right)$ of $0^{\circ}(\mathrm{emission}$ along the surface normal). For (b) and (c) the best fits (light blue markers) to the experimental data (solid black lines) are also displayed, achieved with a combination of GL (broken red lines), LF (broken red lines), and Shirley-type (broken grey lines) functions. Peak labels are explained in the main text. Please click here to view a larger version of this figure. 

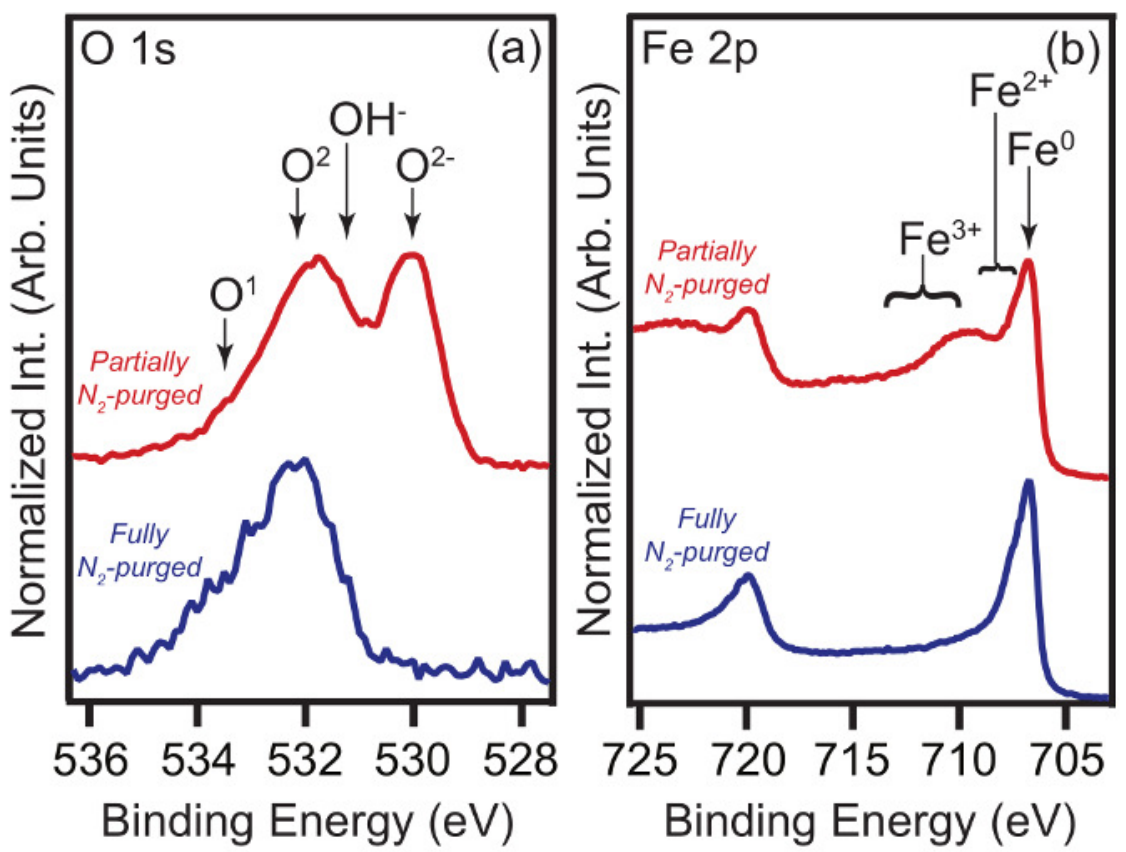

Figure 2. Impact of post immersion oxidation on XPS spectra. (a) O 1s and (b) Fe $2 p$ XPS spectra. Data were acquired from carbon steel samples that had been immersed in $1 \mathrm{M} \mathrm{HCl}+2 \mathrm{mM} \mathrm{MBI}$ solutions $(n \%=99 \%)$ for $4 \mathrm{~h}$. Spectra were acquired at $\theta_{E}=0^{\circ}$. In each panel, the lower (upper) spectrum is from a sample transferred through a fully (partially) $\mathrm{N}_{2}$-purged glove box. Peak labels are explained in the main text. (Modified version of Fig. 5 in Ref. 9.) Please click here to view a larger version of this figure.

\section{Discussion}

XPS spectra displayed in Figures 1 and 2 clearly demonstrate that the inert atmosphere employed during sample transfer is essential to avoid post immersion oxidation of these carbon-steel/organic-Cl interfaces. On this basis, results from other similar XPS studies (e.g. ${ }^{18,19}$ ), which involved exposure of the inhibited substrate to the ambient laboratory atmosphere, should be critically re-evaluated, as the interface chemistry may have been modified through oxidation. It should be noted that that there is no reason to assume that all organic-Cls adsorb onto oxide/ hydroxide free surfaces in acidic solution. In some cases, such phases may indeed facilitate the organic-Cl surface binding. Distinguishing this scenario from post immersion oxidation is not so straightforward. One possible solution is to acquire XPS data from a reference inhibited interface (e.g. $1 \mathrm{M} \mathrm{HCl}+2 \mathrm{mM} \mathrm{MBI})$ in tandem with the sample of interest to check that post immersion oxidation is not an issue.

To ensure a successful outcome to the sample transfer procedure, it is essential that the glove box is fully purged with inert gas $\left(\mathrm{N}_{2} / \mathrm{Ar}\right)$ i.e. the $\mathrm{O}_{2}$ concentration in the glove box is minimized. Care should be taken to verify that all seals on the glove box ports/entry points are correctly formed, including the seal between the glove box and XPS load-lock flange. Ideally, an in situ sensor should be used to directly monitor the $\mathrm{O}_{2}$ concentration, although it is not essential, as demonstrated by our own work. As indicated in step 3.3.2, we normally use a relative humidity sensor as a guide for when to undertake a sample transfer.

One further potential issue with the glove box environment is the presence of volatile solution components, which can contaminate the sample surface following removal from solution and prior to insertion into the load-lock. For example, the presence of $1 \mathrm{M} \mathrm{HCl}$ solution in the glove box leads to evolution of $\mathrm{HCl}$ vapor, which can react with carbon-steel samples leading to adventitious $\mathrm{Cl}$ signal in the XPS data. To minimize the likelihood of such contamination being significant, small volumes of $\mathrm{HCl}$ solution should be employed and sample transfer should be completed as quickly as possible. On this basis, as indicated in the Protocol, typically only one beaker/sample is inserted into the glove box for sample transfer at any one time. In addition, one should minimize the surface area of the $\mathrm{HCl}$ solution, as well cover the beaker following sample emersion. $\mathrm{Na}_{2} \mathrm{CO}_{3}$ powder (step 3.1.4) is inserted into the glove box in an attempt to control the amount of $\mathrm{HCl}$ vapor. In addition, it can be used to clean up any acidic solution spillages.

Besides careful control of the glove box environment, sample handling is also critical to the integrity of acquired XPS spectra. One should not acquire XPS data from any surface that has come into contact with any solid object, e.g. tweezers or glove. Furthermore, upon removal of a sample from solution, it should be immediately blown dry with inert gas (step 3.2.5). This procedure is undertaken to prevent evaporation and subsequent physical deposition of solution components onto the sample surface, which may lead to misinterpretation of data. As an additional precaution, one can also consider replacing the nitrile gloves (step 3.2.3) with a fresh pair for transferring the sample bar to the load-lock chamber, i.e. prior to step 3.1.10.

Finally, given the effectiveness of the approach outlined here, we expect it to be applied to other topics in corrosion (i.e. in addition to corrosion inhibition), where acquisition of XPS data from air-sensitive interfaces would add to understanding. Moreover, such an approach should be considered in other fields where XPS measurements are undertaken from air-sensitive interfaces formed in a fluid environment. Clearly, this procedure is not restricted to XPS, but could also be applied to any other UHV-based measurement from a surface that has previously been immersed in a liquid, e.g. scanning probe microscopy. 


\section{Disclosures}

The authors declare that they have no competing financial interests.

\section{Acknowledgements}

The work was supported by AkzoNobel through a collaboration agreement with The University of Manchester. PMG is grateful to the Fondo Sectorial CONACYT - SENER Hidrocarburos and the Instituto Mexicano de Peteróleo for financial support. TB thanks Mellitah Oil \& Gas Companyfor funding her studentship. KK acknowledges financial support from the EPSRC (EP/L01680X/1) through the Materials for Demanding Environments Centre for Doctoral Training." PAL thanks CONACYT for financially supporting her stay in Manchester. Finally, all of the authors acknowledge Ben Spencer for his technical support and advice.

\section{References}

1. Sastri, V. S. Corrosion Inhibitors: Principles and Applications. Wiley, Chichester, (1998).

2. Lindsay, R., \& Lyon, S. B. Introduction to Control of Corrosion by Environmental Control. In: Shreir's Corrosion, Vol. 4, Management and Control of Corrosion. Cottis, R.A., et al., eds., pp. 2891-2899, Elsevier, Amsterdam (2010).

3. Oguzie, E. E., Li, Y., Wang, S. G., \& Wang, F. Understanding corrosion inhibition mechanisms - Experimental and theoretical approach. RSC Advances. 1 (5), 866-873 (2011).

4. Kokalj, A., Peljhan, S., Finšgar, M., \& Milošev, I. What determines the inhibition effectiveness of ATA, BTAH, and BTAOH corrosion inhibitors on copper? Journal of the American Chemical Society. 132 (46), 16657-16668 (2010).

5. Kokalj, A. Formation and structure of inhibitive molecular film of imidazole on iron surface. Corrosion Science. 68 195-203 (2013).

6. Briggs, D., \& Seah, M. P. Practical surface analysis: by auger and x-ray photo-electron spectroscopy. vol. 1, second ed., Wiley, Chichester (1996).

7. Finšgar, M., \& Jackson, J. Application of corrosion inhibitors for steels in acidic media for the oil and gas industry: A review. Corrosion Science. 86 17-41(2014).

8. Finšgar, M. 2-Mercaptobenzimidazole as a copper corrosion inhibitor: Part II. Surface analysis using X-ray photoelectron spectroscopy Corrosion Science. 72 90-98 (2013).

9. Morales-Gil, P., Walczak, M. S., Cottis, R. A., Romero, J. M., \& Lindsay, R. Corrosion inhibitor binding in an acidic medium: Interaction of 2mercaptobenizmidazole with carbon-steel in hydrochloric acid. Corrosion Science. 85 109-114 (2014).

10. Olivares-Xometl, O., Likhanova, N. V., Martínez-Palou, R., \& Domínguez-Aguilar, M. A. Electrochemistry and XPS study of an imidazoline as corrosion inhibitor of mild steel in an acidic environment. Materials and Corrosion. 60 (1), 14-21 (2009).

11. Flores, E. A. et al. Sodium phthalamates as corrosion inhibitors for carbon steel in aqueous hydrochloric acid solution. Corrosion Science. $\mathbf{5 3}$ (12), 3899-3913 (2011).

12. Zarrok, H. et al. Corrosion control of carbon steel in phosphoric acid by purpald - Weight loss, electrochemical and XPS studies. Corrosion Science. 64 243-252 (2012).

13. Morales-Gil, P. et al. Corrosion inhibition of carbon-steel with 2-mercaptobenzimidazole in hydrochloric acid. Corrosion Science. 101 47-55 (2015).

14. Popova, A., Christov, M., Raicheva, S., \& Sokolova, E. Adsorption and inhibitive properties of benzimidazole derivatives in acid mild steel corrosion. Corrosion Science. 46 (6), 1333-1350 (2004).

15. Gupta, R. P., \& Sen, S. K. Calculation of multiplet structure of core p -vacancy levels. Il. Physical Review B. 12 (1), 15-19 (1975).

16. Shirley, D. A. High-resolution x-ray photoemission spectrum of the valence bands of gold. Physical Review B. 5 (12), $4709-4714$ (1972).

17. Al-Refaie, A. A., Walton, J., Cottis, R. A., \& Lindsay, R. Photoelectron spectroscopy study of the inhibition of mild steel corrosion by molybdate and nitrite anions. Corrosion Science. 52 (2), 422-428 (2010).

18. Liu, X. et al. The study of self-assembled films of triazole on iron electrodes using electrochemical methods, XPS, SEM and molecular simulation. Electrochemistry Communications. 9 (4), 813-819 (2007).

19. Zhang, $\mathrm{H}$. et al. Corrosion inhibition of mild steel in hydrochloric acid solution by quaternary ammonium salt derivatives of corn stalk polysaccharide (QAPS). Desalination. 372 57-66 (2015). 